\title{
Eindrücke aus der Citrus-Ausstellung 2019
}

\section{Hilke Steinecke}

Das Sommerhalbjahr 2019 war im Palmengarten ganz dem Thema "Citrus" gewidmet. Die Informationsausstellung „Citrus - zwischen Glanz und Gloria" wurde vom 23. Mai bis 22. September 2019 in der Galerie West am Palmenhaus gezeigt.

Die Ausstellung war bereits einige Jahre zuvor für das Jahr 2019 eingeplant. In dieser Zeit konnte die noch recht junge Citrus-Sammlung in der Gärtnerei des Palmengartens weiter ausgebaut und auf die Bedürfnisse der Ausstellung zugeschnitten werden. Rund 100 Exemplare in etwa 70 verschiedenen Arten bzw. Sorten konnten schließlich die Ausstellung bereichern und die Vielfalt der Gattung Citrus veranschaulichen.

Neben der Biologie von Zitruspflanzen, dem besonderen Bau und der Vielfalt der Früchte sowie

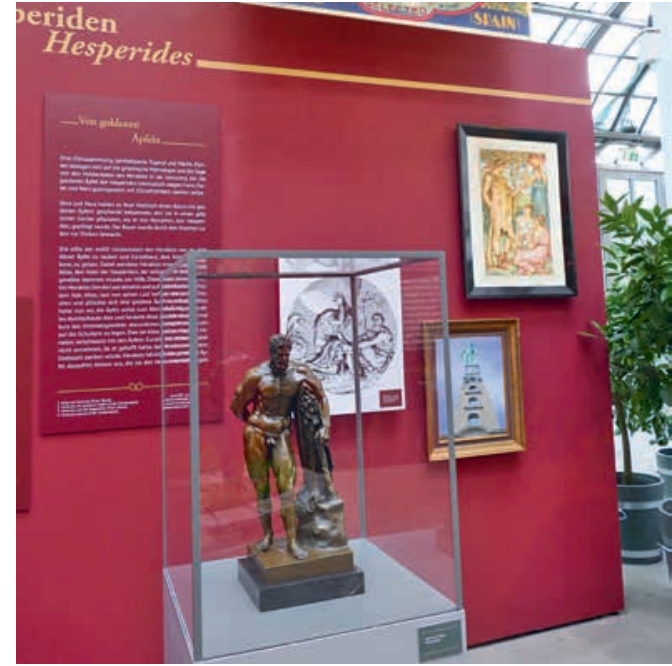

Abb. 2: Infowand zur Symbolik von Citrus, davor eine Reproduktion des Herkules Farnese. (Foto: H. Steinecke)

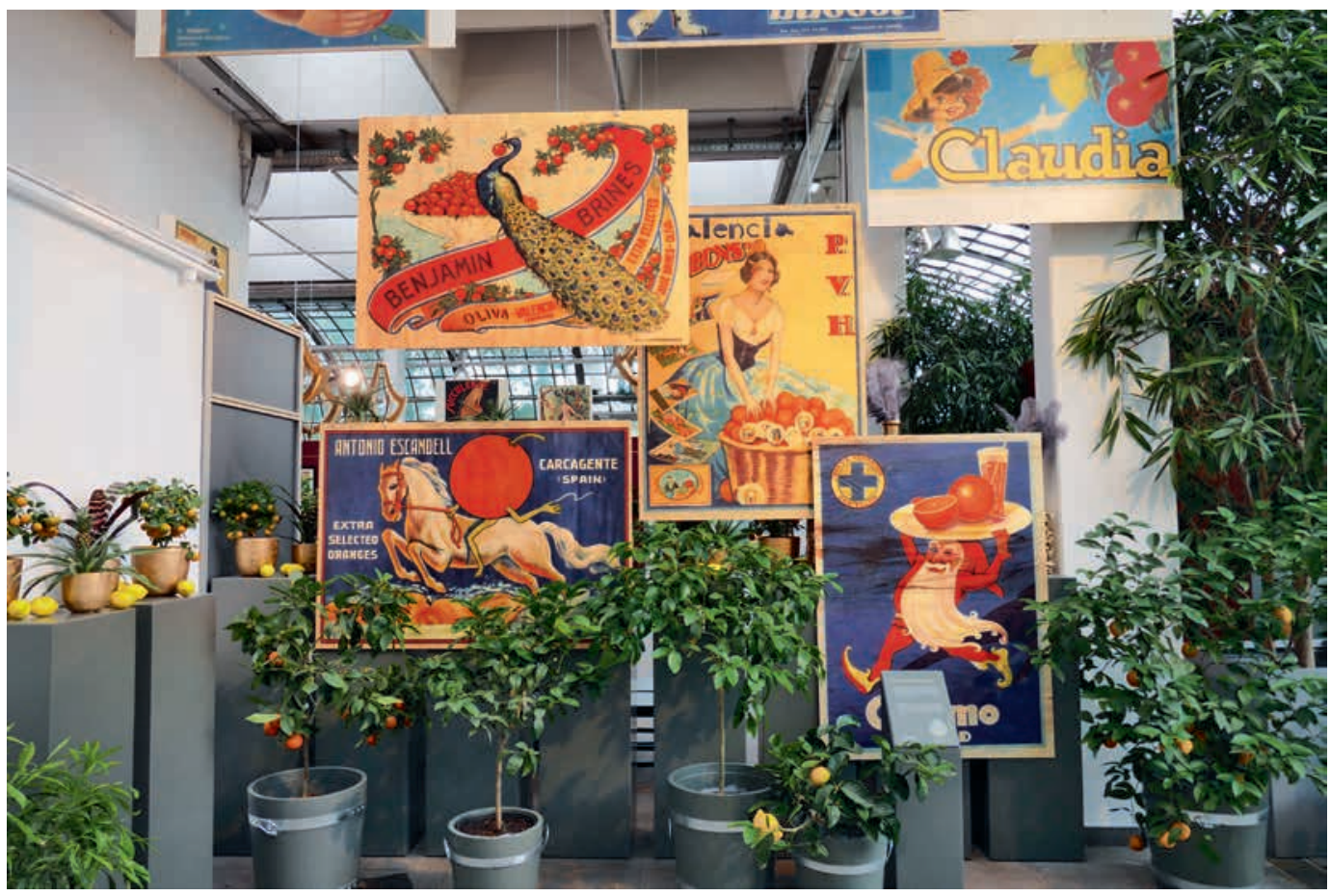

Abb. 1: Im Eingangsbereich der Ausstellung waren verschiedene Orangenkistenplakate zu sehen. Dahinter befand sich der Stand des Reformhauses Freya mit kleiner Café-Ecke. (Foto: H. Steinecke) 


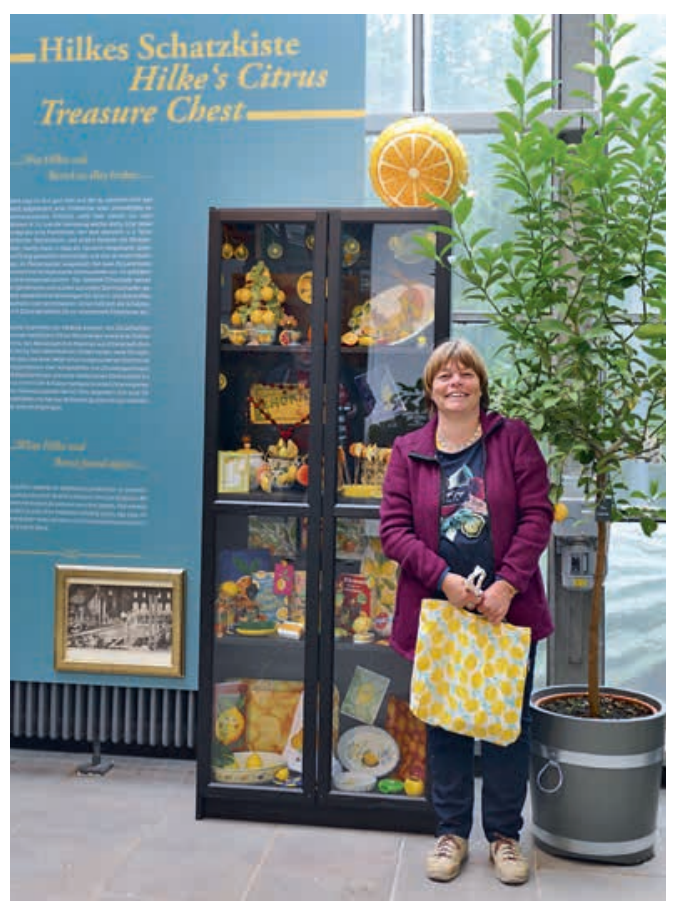

Abb. 3: Eine „Schatzkiste“ mit diversen Fundstücken zum Thema Citrus zur Veranschaulichung der vielseitigen Verwendung der Früchte. (Foto: H. Steinecke)

der Nutzung standen vor allem kulturhistorische Aspekte im Vordergrund. Wo kommen die Zitruspflanzen ursprünglich her und welches sind die drei Ursprungsarten? Wie kamen sie zu uns, wie wurden sie in Südeuropa und später auch nördlich der Alpen kultiviert? Wie sahen die Orangerien aus? Was müssen Gärtnerinnen und Gärtner beachten, wenn Zitruspflanzen gut gedeihen sollen? Die früher sehr wertvollen und exotischen Zitruspflanzen mitsamt ihren Früchten waren früher vor allem Adeligen und Reichen zugänglich. Sie wurden hoch geschätzt und hatten hohen Symbolwert. Dementsprechend gab es auch einen Exkurs in die Symbolik von Citrus. Dabei durfte die griechische Sage von Herakles und dem Raub der drei goldenen Äpfel der Hesperiden, die später mit Zitrusfrüchten gleichgesetzt wurden, nicht fehlen. Herakles erzählte an einer Station auf Knopfdruck seine elfte Heldentat sogar auf Hessisch. Hingucker waren in der Ausstellung auch Reproduktionen der berühmten Kupferstiche der Nürnberger Hesperiden.
Zitruspflänzchen gibt es mittlerweile in fast jedem Discounter zu kaufen, und sie sind mittlerweile beliebte Zimmerpflanzen. Doch nicht bei jedem gedeihen sie immer problemlos. Deshalb wurden auch hilfreiche Infos rund um die Pflege von Zitruspflanzen gegeben. Ein Pflanztisch und ein Einblick in die ideale Schichtung des Substrates mit einer Drainageschicht sorgten für ein passendes Ambiente.

Am Ende des Ausstellungsrundgangs wurden unzählige Citrus-Produkte gezeigt, auch wenn der Zitrusduft vieler Haushaltsreiniger nicht mehr aus den entsprechenden echten Früchten gewonnen wird, sondern synthetisch hergestellt wird. Hinterfragt werden sollte auch, woher die vielen Orangen für den Saft oder den Frischverzehr kommen. Die meisten Saftorangen werden in Brasilien in riesigen Monokulturen angebaut und mit reichlich Pestiziden behandelt. Im Vergleich zur Weltproduktion ist der Bioanbau sehr gering. Stellvertretend für die wirtschaftliche Bedeutung der Zitrusfrüchte und den Handel vor allem mit Orangen waren über die Ausstellung verteilt vergrößerte Reproduktionen von Orangenkistenplakaten zu sehen. Die teilweise sehr witzigen und an Comiczeichnungen erinnernden Motive fanden vor allem in den 1950er

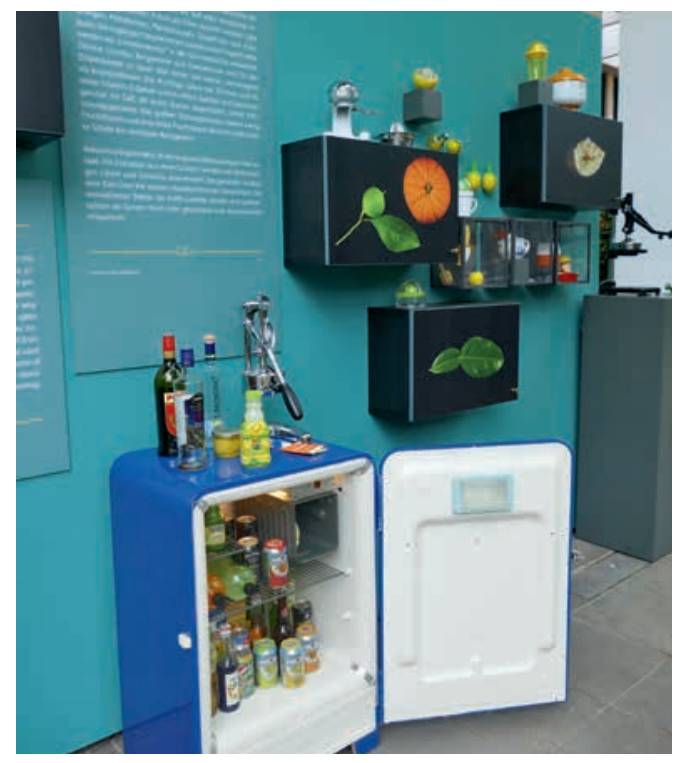

Abb. 4: Kühlschrank mit diversen Citrus-Getränken sowie verschiedene Modelle von Zitronenpressen.

(Foto: H. Steinecke) 


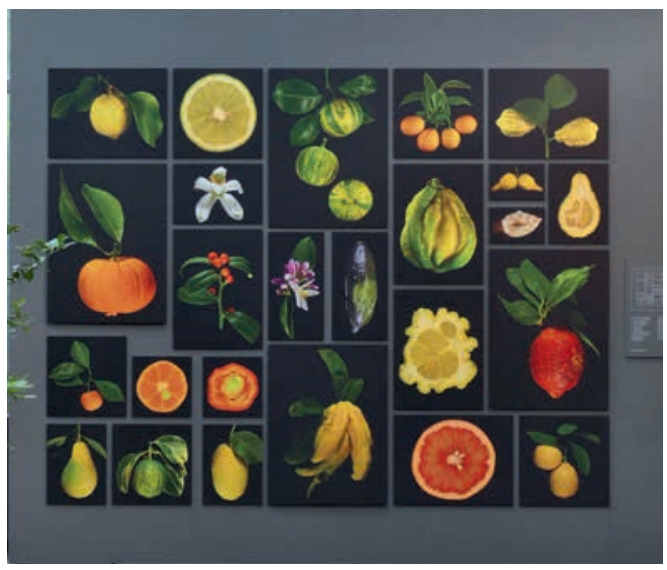

Abb. 5: Einen Eindruck von der Vielfalt der Früchte innerhalb der Gattung Citrus vermittelten die brillanten Fotos von Jennifer Markwirth. (Foto: H. Steinecke)

Jahren Verwendung, als die Holztransportkisten mit Orangen für den Kunden durch die Plakate attraktiver gemacht werden sollten.

Das Besondere an den Informationsausstellungen im Palmengarten ist die Kombination aus Informationen, attraktiven Exponaten und vor allem den zum Thema passenden Pflanzen. Diesmal konnten wir aus dem Vollen schöpfen. Alle in der Ausstellung gezeigten Pflanzen stammten aus der Sammlung der Palmengarten-Gärtnerei. Die Pflanzen hatten während der gesamten Ausstellungsdauer Blüten und trugen Früchte. Das Spektrum reichte von der Mini-Kumquat ( $C$. hindsii) mit etwa erbsenkleinen Früchten bis hin zu großfrüchtigen Exemplaren wie Pampelmuse und Zitronat-Zitrone. Großes Interesse fanden die Fingerlimetten (Citrus australasica), deren Saftschläuche perlenartig kugelig sind und an Kaviar erinnern. Sie werden auch Limettenkaviar bezeichnet und in der Gourmetküche verwendet. Immer wieder staunten Besucherinnen und Besucher der Ausstellung über die bizarren, handförmig geteilten Früchte von „BudDHAs Hand“.

Die Vielfalt der Fruchtformen z. T. alter Sorten wurde nicht nur anhand der lebenden Pflanzen präsentiert. Viele Früchte aus eigener Palmengarten-Ernte begeisterten auch in Form von brillanten Fotos. Fehlen durfte dabei auch nicht eine Kuriosität, nämlich eine Zitrone mit außen roter Schale und innen normal gelbem Fruchtfleisch.

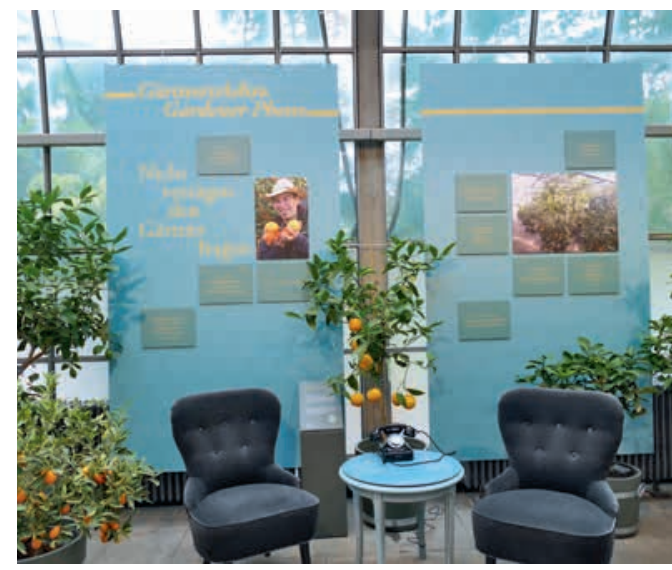

Abb. 6: Unser Citrus-Gärtner Alexander Becker gab auf einem Ausstellungs-Telefon Tipps zur Pflege von CitrusPflanzen. (Foto: H. Steinecke)

Die Ausstellung mitsamt dem Rahmenprogramm wurde sehr gut angenommen, auch das ausliegende Zitrusquiz fand großen Zuspruch.

Die Ausstellung war nicht als Wanderaustellung konzipiert und ist in der gezeigten Form nicht ausleihbar. Erhältlich sind aber noch Restbestände des Sonderheftes („Citrus“, Nr. 51) mit ausführlichen Texten zum Inhalt der Ausstellung. Druckvorlagen für die Citrus-Informationen können bei Bedarf beim Palmengarten angefragt werden.

\section{Anschrift der Autorin}

Dr. Hilke Steinecke, Palmengarten Frankfurt, Siesmayerstraße 61, 60323 Frankfurt, E-Mail: hilke.steinecke@stadt-frankfurt.de

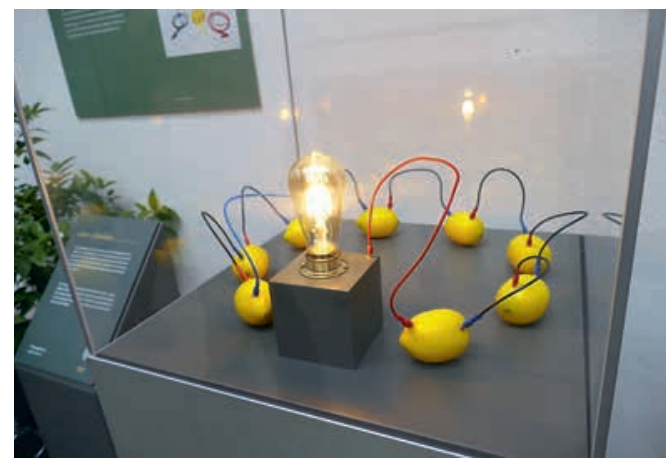

Abb. 7: Für „Erleuchtung“ sorgte in der Experimente-Ecke ein Modell der „Zitronenbatterie“. (Foto: H. Steinecke) 